\title{
Preparation of Associative Polyurethane Thickener and Its Thickening Mechanism Research
}

\author{
Gao Nan, ${ }^{1,2}$ Zhang Zhuo, ${ }^{2}$ and Dong Qingzhi ${ }^{1}$ \\ ${ }^{1}$ East China University of Science and Technology, Shanghai 200237, China \\ ${ }^{2}$ Shanghai Institute of Technology, Shanghai 200235, China \\ Correspondence should be addressed to Gao Nan; gaonanxf@sit.edu.cn
}

Received 24 July 2015; Revised 6 September 2015; Accepted 17 September 2015

Academic Editor: John Zhanhu Guo

Copyright (c) 2015 Gao Nan et al. This is an open access article distributed under the Creative Commons Attribution License, which permits unrestricted use, distribution, and reproduction in any medium, provided the original work is properly cited.

\begin{abstract}
Associative polyurethane (PU) thickener has been synthesized by preparing the prepolymer with the reaction of polyethylene glycol (PEG) and isophorone diisocyanate (IPDI), which then end-capping with long-chain alkanol. The synthesis process, as well as hydrophilic chain length, theoretical molecular weight, solvent ratio, and thickener percentages, has been researched. The results shows that it reaches the upmost thickening effect when the theoretical molecular weight is under 20000, soft-segment length is under 4000 , solvent ratio is $1: 1$, and thickener percentages are controlled at $10 \%$. Furthermore, thickening mechanism of PU thickener has been analyzed detailedly through the measurement of the critical micelle concentration (CMC) of PU thickener and analysis of the influence of PU thickener on the particle size and morphology of PU dispersions. It has been observed from the scanning electron microscopy (SEM) that the PU aqueous dispersions produce a certain degree of flocculation when the PU thickener was added, and this flocculation structure has been proved to be a thixotropic structure through the characterization of the change of particle size before and after the thickener is introduced into the PU aqueous dispersions. The CMC measurement results present that the thickening effect will be apparent when the concentration is controlled in a low range.
\end{abstract}

\section{Introduction}

In recent years, as the importance of environment protection is more and more valued, waterborne coatings and other coatings with low VOC content have been unprecedentedly developing, among which latex coatings as a representative of the waterborne coatings become an important direction of development. However, there are still various kinds of problems in the development of water-borne coatings. The body of water-borne coatings is the mixture of pigment aqueous dispersion and polymer dispersion; when the effective volume of the dispersed phase is less than $55 \%$, the viscosity of the water-borne coatings is substantially close to the viscosity of water dispersion medium, which is not yet sufficient in meeting its brush ability, so the additives should be added so as to improve the viscosity of the emulsion. Based on such demands, aqueous thickener has emerged. Thickener is a rheological additive; the addition of thickener will not only thicken the waterborne coatings but also improve their mechanical properties and physicochemical stability [1-3].
Associative PU thickeners are applied more and more extensively in all the waterborne thickeners because of their excellent leveling and thickening effect $[4,5]$. Associative PU thickeners have become one of the most important research areas in the additives of waterborne coatings. In addition, the molecular structure and thickening mechanisms of associative polyurethane thickeners are completely different from the traditional thickeners, and their rheological characteristics also exhibited different characteristics. The relative study has become the focus in the research of coating additives [6-9]. Nonetheless, at present, there is not enough study in this field; the present research is not intensive and systematic, which is only limited to study the thickening effect on the viscosity and film formation in thickening system.

In this paper, the effect of the addition of PU thickener on the particle size and morphology of the aqueous dispersions has been studied and the thickening mechanism of PU thickener has been analyzed detailedly on the basis of the synthesis of waterborne associative polyurethane thickener. 


\section{Experimental}

2.1. Materials. The materials were as follows: PEG1500, PEG4000, and PEG6000 (industrial grade, Shanghai resins plant) and IPDI (BASF); 16 alcohol, n-octanol, nonylphenol, dibutyltin February cinnamate, and stannous octanoate, all analytical grade; PU water dispersions (homemade).

2.2. Laboratory Procedures. The PEG and seal end agent were introduced into a four-necked vessel with reflux condenser, stirrer, and thermometer and then heated to $100^{\circ} \mathrm{C}$ and dehydrated under vacuum for $2 \mathrm{~h}$. Then the vessel was cooled to $70^{\circ} \mathrm{C}$ for the dropping of IPDI with high-speed stirring. When the dropping of IPDI was finished, the mixture was heated up to $80^{\circ} \mathrm{C}$ for the addition of catalyst. And then the reaction system was heated to $120^{\circ} \mathrm{C}$ and stirred for 3 minutes. Finally, the reaction was stopped and the material was moved to the oven preheated to $100^{\circ} \mathrm{C}$, and then the yellow solid products were obtained after the materials were dried for 6 hours.

2.3. Preparation of Thickening Agent. The yellow solid was dissolved in solvents with appropriate proportion, and then they were put into a water bath with constant temperature for $10 \mathrm{~min}$. The thickener products were obtained after stirring for $2 \mathrm{~min}$.

\subsection{Characterization}

2.4.1. Measurement of Particle Size and Particle Size Distribution. The particle size and its distribution of the obtained dispersion were measured by a LS 230 laser particle sizer produced by British MALVEN Company and the measuring range was $0.02 \mu \mathrm{m} \sim 2 \mathrm{~mm}$.

2.4.2. Viscosity Tests. The viscosity of the samples was measured with DNJ-9S.

2.4.3. Measurement of Molecular Weight. The molecular weight was determined by Waters 1515 gel permeation chromatograph. The separation column is 3-series cross-linked polystyrene column; The mobile phase was tetrahydrofuran (THF) with the flow rate of $1.0 \mathrm{~mL} / \mathrm{min}^{-1}$; the detector was Waters 2414 differential refractive index detector.

2.4.4. SEM Observation. PU water dispersions were diluted, and a drop of water dispersion was dropped in sample table with conductive adhesive. The morphology of water dispersion was observed under the scanning electron microscopy (SEM) after drying, surface spray gold.

2.4.5. UV-Visible Spectra Determination. UV method was used to determine the CMC of the PU thickener at $350 \mathrm{~nm}$.

\section{Results and Discussion}

3.1. Influences of Theoretical Molecular Weight on the Thickening Effect. Figure 1 shows the effects of different theoretical

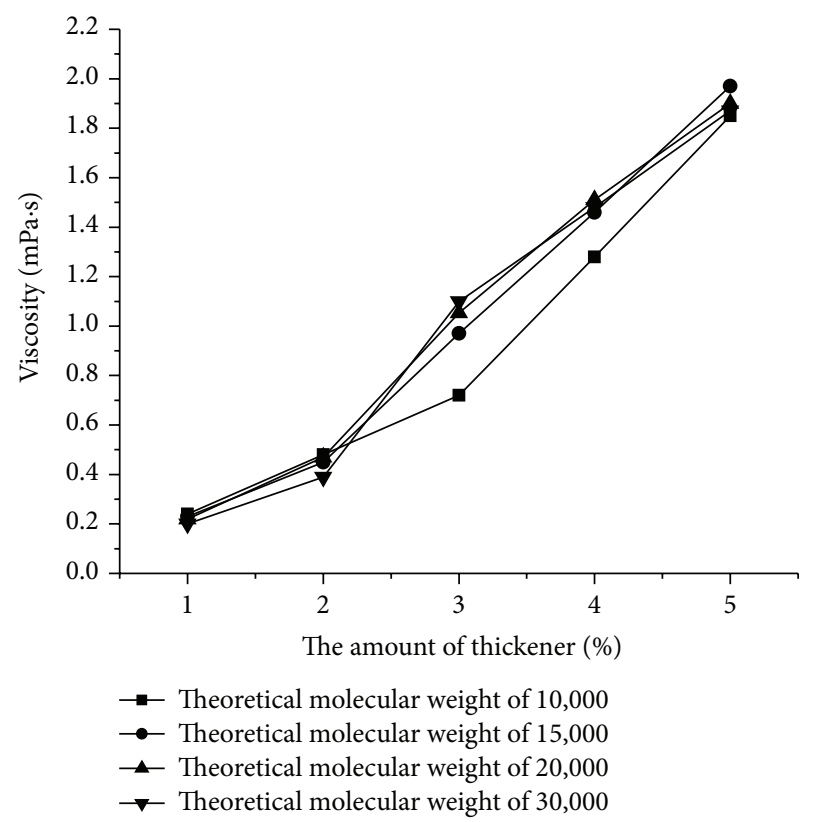

FIGURE 1: Thickener with different ideal molecular weight versus viscosity of PU dispersions.

molecular weight on the thickening effect of PU dispersions. The results indicate that the thickening effect has some improvement with the increase of theoretical molecular weight (10 20 thousand). The reason may be that the hydrophobic segment and hydrophilic segment have a certain degree of growth with the increase in molecular weight, which is more conducive to make the thickener molecule to format network in the system; but with the further increase in molecular weight (20 30 thousand), the thickening effect has no apparent change. As the same time, the adhesion level of the reactor increases with the yield decreased. Taking into account the thickening effect and yield, the theoretical molecular weight of 20,000 is selected.

Figure 2 is the theory of molecular weight of 20,000 thickener GPC curves. It can be seen that the GPC curve has an obvious outflow peak, the weight-average molecular weight of polymer is 19,016 and molecular weight distribution is 1.6 according to the outflow time, and the results show that the actual measured molecular weight is close to the molecular weight in theory.

3.2. Influences of Soft-Segment Length on the Thickening Effect. Figure 3 shows the effect of thickeners prepared by different hydrophilic length segment polyol on the thickening efficiency of the aqueous dispersion. From Figure 3, the thickeners with different hydrophilic length segment are all proven to have a certain thickening effect. And the thickening effect of the thickeners prepared by PEG4000 is improved to some extent compared to thickeners prepared by PEG1500; this may be because the growth of the hydrophilic segment of the thickener molecule chain is conducive to the uniform dispersion of the thickener molecules in the system. But with 


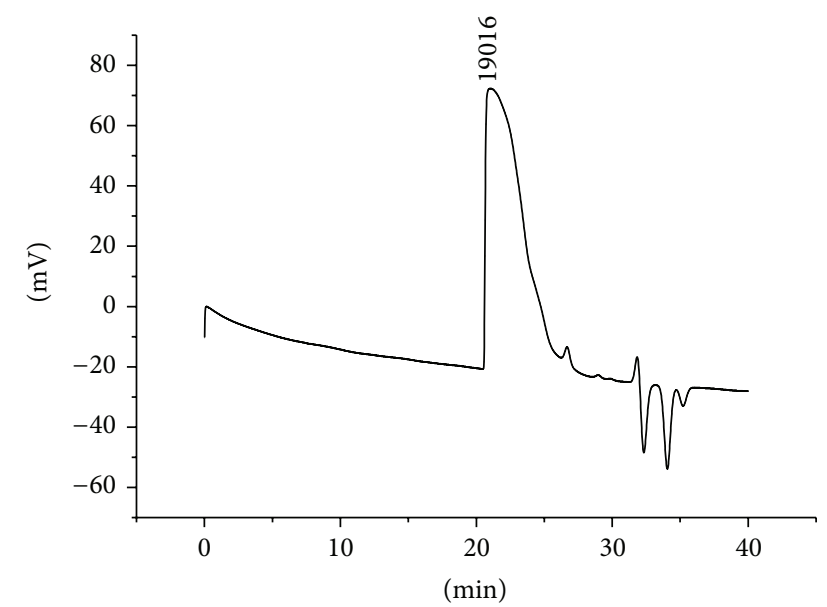

FIGURE 2: GPC graph of PU dispersions (ideal molecular weight of 2000).

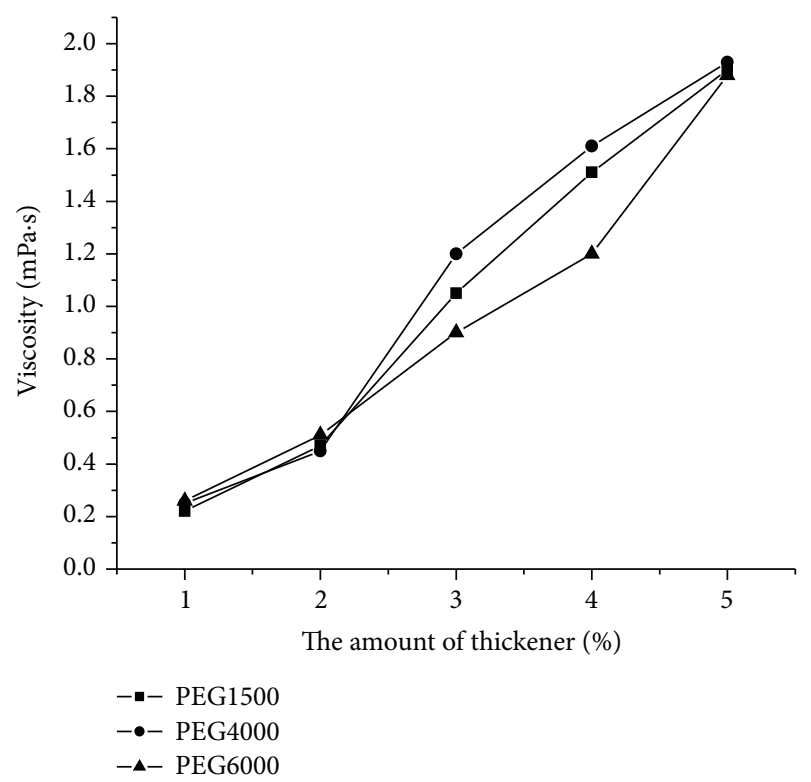

Note: The $10 \%$ aqueous solution; theoretical molecular weight of 20,000

FIgURE 3: Thickener with different PEG versus viscosity of PU dispersions.

the further increase in the hydrophilic length segment (PEG, from 4000 to 6000), the existing thickening effect is not only maintained but also decreased, which may be due to the fact that hydrophilic chain paragraph also makes a further increase in the difficulty of IPDI dispersion in the reaction system, leads to the appearance of topical gel, and has a great influence on the thickening effect. Hydrophilic segment is not the longer the better. The appropriate choice of the length of the hydrophilic segment should take into account two aspects including the thickening effect and polymerization controllable.

3.3. Influences of Solvent Ratio on the Thickening Effect. Figure 4 shows the curves of different ratio of solvent on

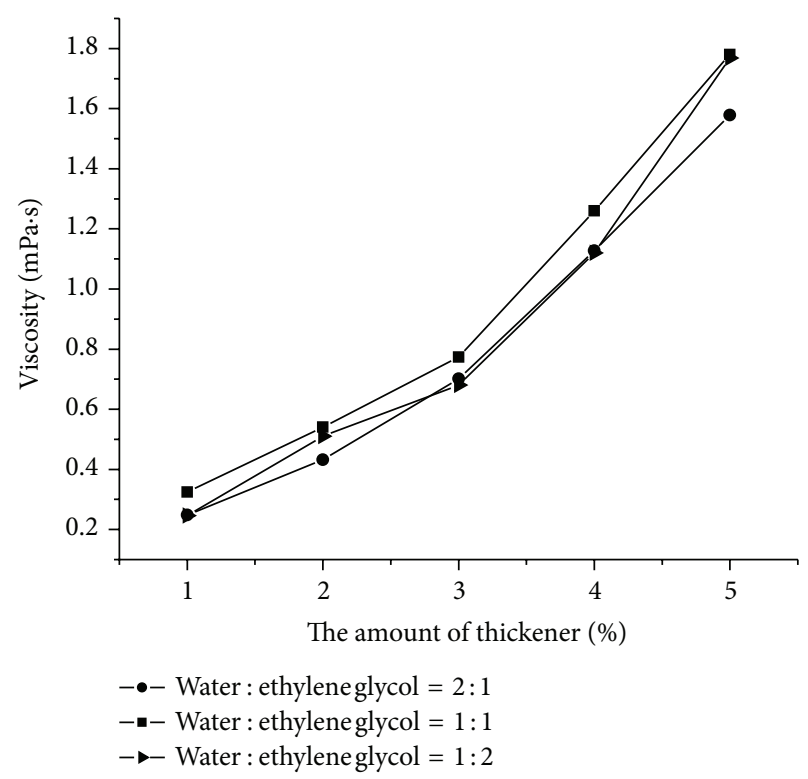

Note: thickener concentration of $20 \%$

FIGURE 4: Thickener with different solvent ratio versus viscosity of PU dispersions.

the thickening effect of thickener. In Figure 4, the thickening effect has some improvement with the increase of ethylene glycol amount (water/ethylene glycol $=2: 1 \sim$ water/ethylene glycol $=1: 1)$. This may due to the fact that the increase of ethylene glycol amount is conducive to make the thickeners uniformly dispersed in the system and thereby has the better thickening effect, but with the further increase of ethylene glycol amount (water/ethylene glycol $=1: 1 \sim$ water/ethylene glycol $=1: 2$ ), viscosity there has a downward trend; this may be because the increase of ethylene glycol amount is in favor of the better distribution of thickener in system, but simultaneously, the excessive amount of ethylene glycol can reduce the viscosity of the system and increase the leveling of aqueous dispersions to a certain extent. So water/glycol ratio of $1: 1$ was selected as the suitable solvent ratio.

3.4. Influences of Thickener with Different Percentages on the Thickening Effect. Table 1 provides the influences of thickener with different percentages on the thickening effect. By Table 1, the increase of thickener concentration helps to heighten the viscosity of dispersions but also makes the dispersion of thickener harder and appear obviously agglomeration. $10 \%$ of thickener concentration was selected taking into consideration the uniform dispersion of thickener in the aqueous dispersions.

\subsection{Thickening Mechanism Research}

3.5.1. Thickening Schematic Diagram. The mechanism of the traditional thickeners is shown in Figure 5; it can be seen that a three-dimensional network structure is formed by the physical interactions between the thickening molecules and 
TABLE 1: Effect of cross-linking agent on the viscosity of PU dispersions.

\begin{tabular}{|c|c|c|c|c|}
\hline \multirow{2}{*}{$\begin{array}{l}\text { The amount of } \\
\text { thickener (\%) }\end{array}$} & \multicolumn{4}{|c|}{ The concentration of thickener (\%) } \\
\hline & 10 & 20 & 30 & 40 \\
\hline & \multicolumn{4}{|c|}{ Viscosity $(\mathrm{Pa} \cdot \mathrm{s})$} \\
\hline 1 & 0.18 & 0.24 & 0.23 & 0.21 \\
\hline 2 & 0.38 & 0.54 & 0.75 & $>2$ \\
\hline 3 & 0.89 & 1.23 & $>2$ & \\
\hline 4 & 1.32 & $>2$ & & \\
\hline 5 & $>2$ & & & \\
\hline
\end{tabular}

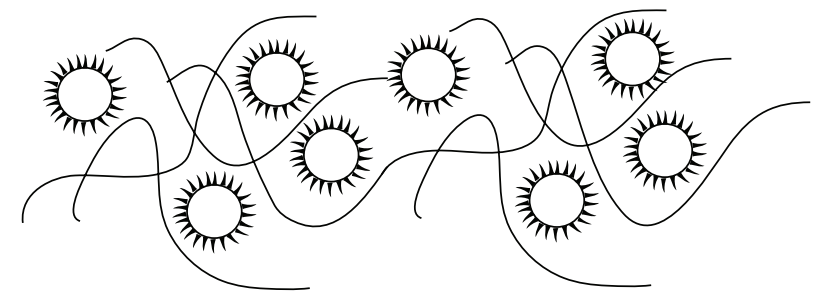

FIGURE 5: Thickening mechanism of traditional thickener.

dispersion medium, which makes the thickening molecules fully stretch so as to achieve the purpose of thickening. Figure 6 shows the thickening mechanism of the associative thickener synthesized in this paper, the thickening mechanism of such thickeners benefits from its more special three-block polymer structure that is "lipophilic-hydrophiliclipophilic" form, and the middle segment is a water-soluble polyethylene glycol chain and the ends are capped by aliphatic hydrocarbon. If the system is waterborne, the concentration of the thickener is relatively large, greater than its critical micelle concentration, and micelles are generated by the association of the lipophilic end groups so as to form the network structure. Water couples with the hydrophilic groups to form a terminal group of micelles and the different micelles can be adsorpted by two lipophilic sides. The thickener molecules form an effective bridge-connection, which makes the polymer particles form association network structure and so the viscosity of the system increases because of the link and winding.

3.5.2. Theoretical Analysis. So as to further understand the thickening mechanism of the associative thickener produced in this experiment, the change in particle size and morphology of the PU dispersions before and after adding the thickener are measured. The results can be seen in the Figures 7,8 , and 9.

Figures 7 and 8 show the changes in particle size of PU aqueous dispersions before and after adding the thickener. The particle size of the aqueous dispersion can be seen as a significant change after adding the thickener (Figure 8(a)). Two peaks appear in Figure 8(a), the position of the first peak is equivalent to that of the peak in Figure 7, and so the first

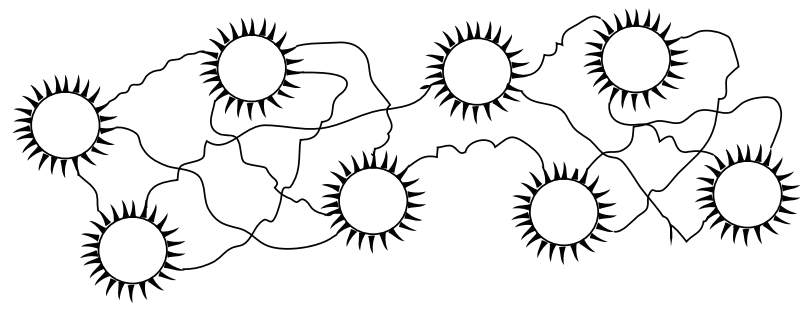

FIGURE 6: Thickening mechanism of associative thickener.

peak can be considered as the original particle size of the PU dispersions, while the second peak can be thought of as the flocculate particles size because of the addition of the associative PU thickener. And it also shows that the particle size is reduced after the ultrasonic dispersion (Figure 8(b)), which also proves that the previous flocculate particles (Figure $8(\mathrm{a})$ ) can be destroyed in the function of the shearing stress (ultrasonic dispersion). Certainly, the flocculate particles still have some residues because the shearing function is not uneven in the dispersion system (Figure 8(b)). The particle size of the aqueous dispersion without adding thickeners has no change before and after adopting the ultrasonic dispersion (Figure 7). This also proves that the network structure is formed and preferably thixotropy of the aqueous dispersions is endowed after adding the thickener.

Figure 9 is a morphological image of the PU aqueous dispersion before and after adding the thickening agent. It can be seen from Figure 9 that the micelles in PU aqueous dispersion produce a certain degree of flocculation. But with reference to the particle size distribution in Figures 7 and 8 , the flocculation structure can be effectively dispersed and destroyed by the outside shearing force, which makes the film forming after thickening ensure not only the viscosity under low shear but also effective leveling under high shear.

In view of the above analysis, the critical micelle concentration (CMC) of the thickener has played a key role, so as to more clearly analyze the thickening mechanism, The CMC of the thickener is measured by the UV-visible spectroscopy, and the experimental results are shown in Figures 10 and 11 and Table 2 . 


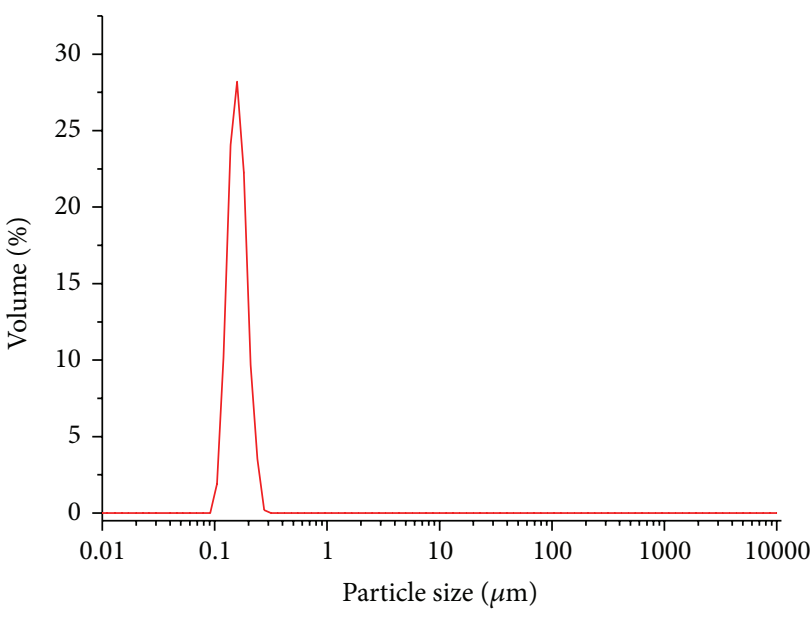

(a) Ordinary dispersion

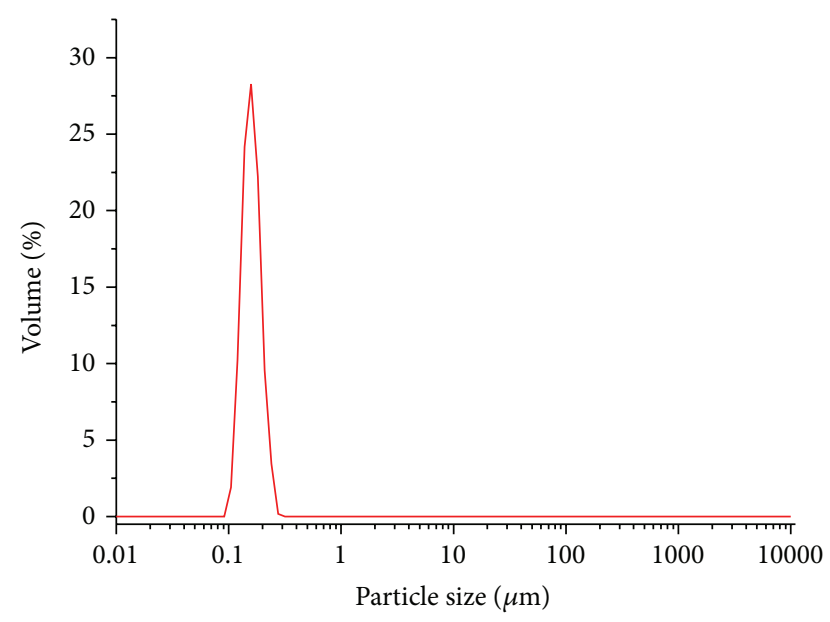

(b) Ultrasonic dispersion

Figure 7: Particle size of PU dispersions without thickener.

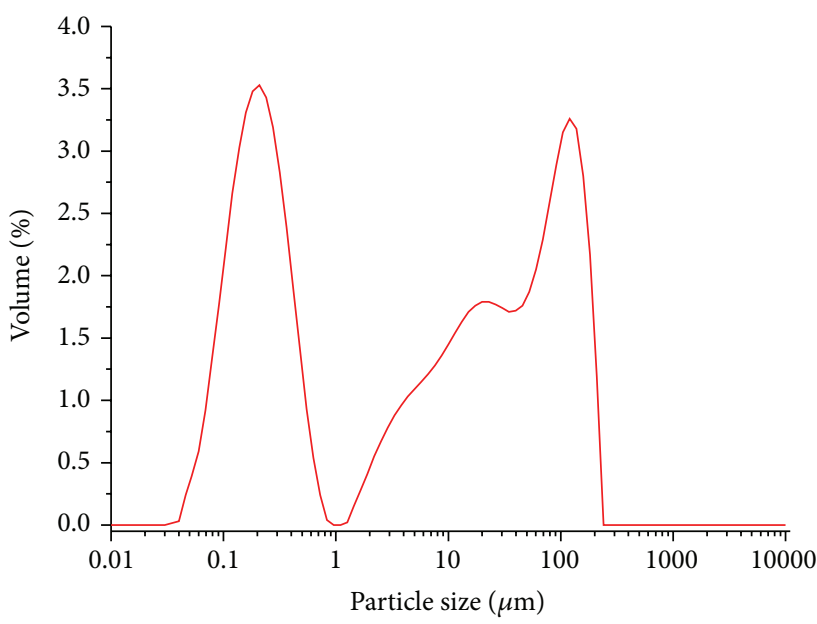

(a) Ordinary dispersion

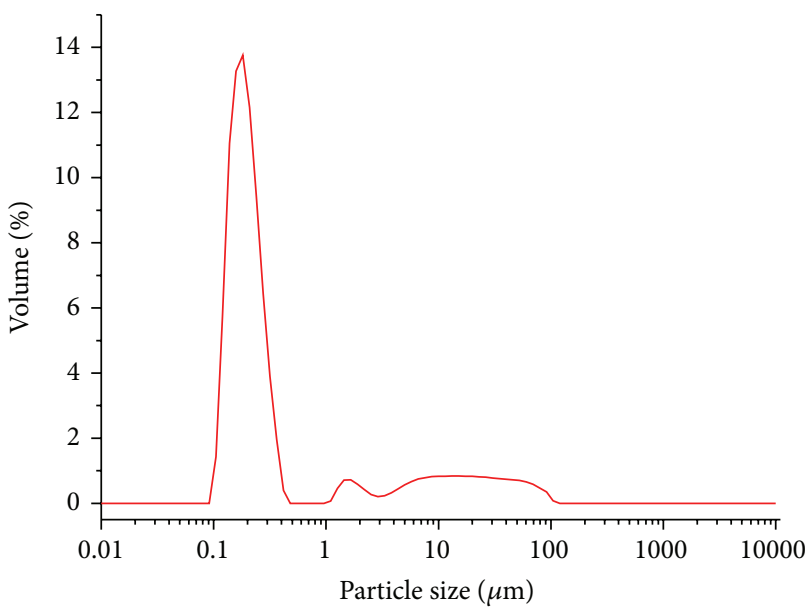

(b) Ultrasonic dispersion

FIGURE 8: Particle size distribution of PU dispersions with thickener.

TABLE 2: CMC value measured by UV-vis.

\begin{tabular}{lc}
\hline Sample number & CMC \\
\hline PU-T1 & $2.5 \times 10^{-4}$ \\
PU-T2 & $1.2 \times 10^{-3}$ \\
PU-T3 & $3.5 \times 10^{-3}$ \\
\hline
\end{tabular}

The absorbance is very low when the concentrations of amphiphilic polyurethane dispersion are lower (Figure 10); the concentration curve is relatively flat which also proves that the micelle has not been formed at this point. However, the absorbance increases sharply when the concentration reaches a certain critical value. The linear fit has been done using the data points which have apparently increased, and the fit line is intended, intersects with the horizontal axis, and obtains a point of intersection which is the CMC of the PU thickeners, as shown in Figure 11. The CMC values obtained are listed in Table 2.

The CMC of nonionic associative polyurethane thickeners is very low through the measurement of three different ABA block-type associative PU thickeners (Table 2), and the formation of the micelles is the main reason of thickening, viscosity increase, and changes in the rheological properties when the shear stress is low. So the thickening effect is apparent when the concentration is controlled in a lower range. And in the process of micelle formation, the hydrophobic segments of the associative thickener form the nucleus layer, and the hydrophilic segment constitutes the shell layer and the molecular chain fully extends at low concentration. With the concentration close to CMC, the hydrophobic segment aggregates, hydrophilic segments are crimped, and the micelles are formed; as a result, the viscosity of the system is effectively increased. 


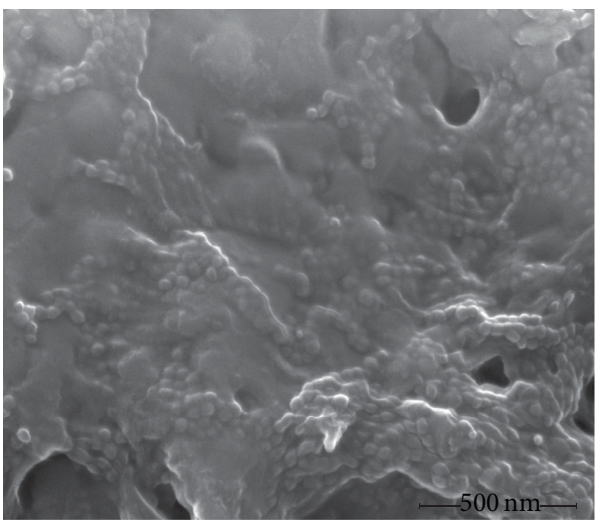

(a) Without thickener

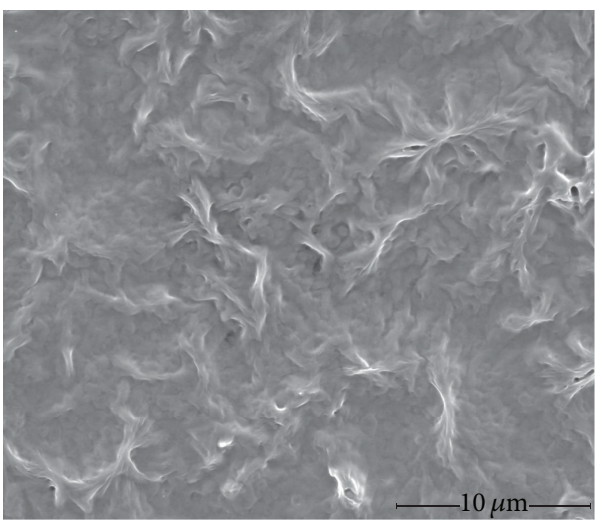

(c) The amount of thickener $2 \%$

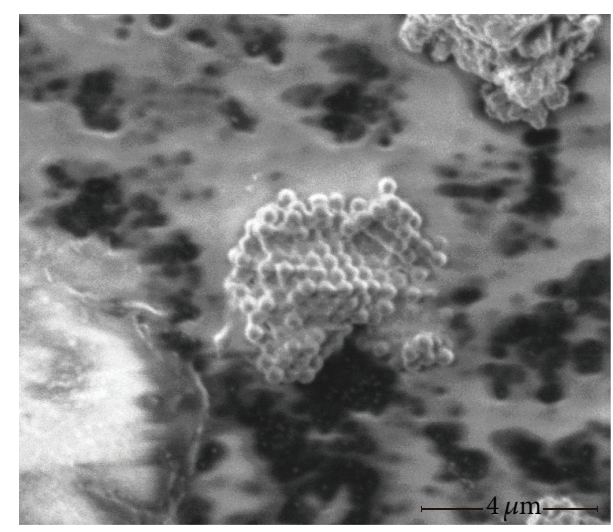

(b) The amount of thickener $1 \%$

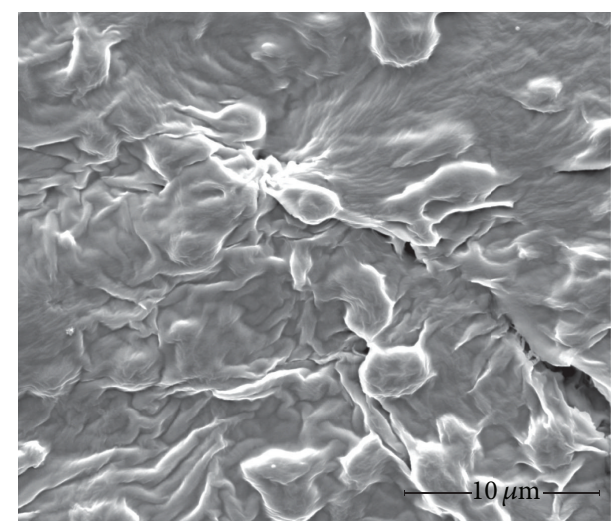

(d) The amount of thickener $3 \%$

FIGURE 9: SEM photograph of PU dispersions with thickener.

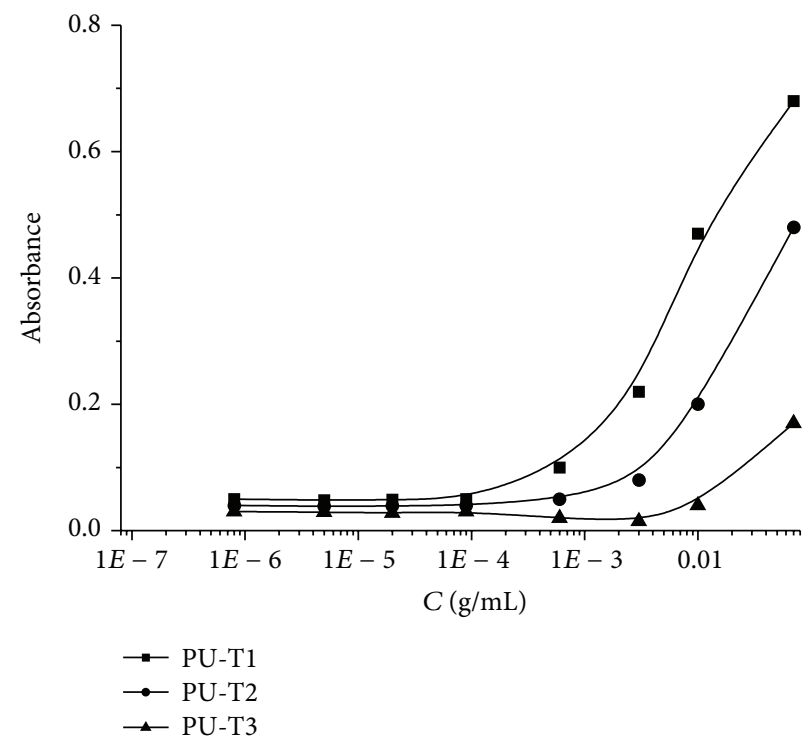

FIGURE 10: Absorbance for thickening agent.

\section{Conclusion}

From the previous results and discussions, different monomer ratio and preparation parameters will have great influence on the thickening effect of PU thickener. The effective
PU thickener with higher hydrophobic segment and tailored molecular weight can be obtained by the selection of suitable monomer ratio, solvent proportion and theoretical molecular weight, and so forth. Moreover, the better understanding of the thickening mechanism prepared in this paper is presented 


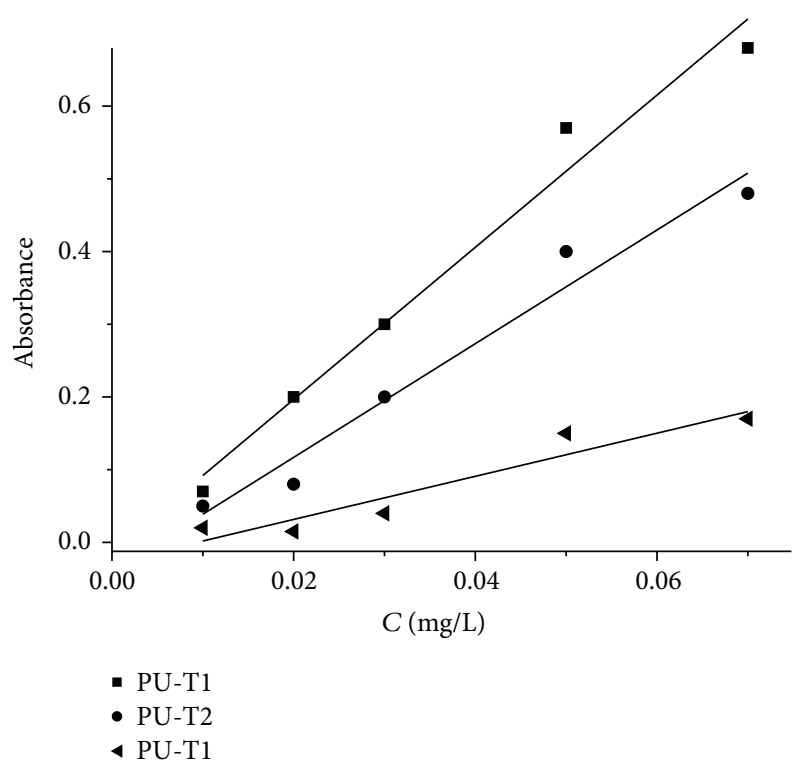

FIGURE 11: Linear fitting curve of the relation between absorption and thickening agent concentration.

by the CMC determination and analysis of the particle size and morphology of the aqueous dispersions thickened by the PU thickener.

\section{Conflict of Interests}

The authors declare that there is no conflict of interests regarding the publication of this paper.

\section{References}

[1] Y. L. Lin, Y. Zhou, C. X. Xu et al., "Study on synthesis and thickening property of hyperbranched waterborne polyurethane," Progress in Organic Coatings, vol. 76, no. 10, pp. 1302-1307, 2013.

[2] M. Kim, Y.-W. Choi, J.-H. Sim, J. Choo, and D. Sohn, "End chain length effect of hydrophobically end-capped poly(ethylene oxide)s on their self-assemblies in solution," The Journal of Physical Chemistry B, vol. 108, no. 24, pp. 8269-8277, 2004.

[3] P. Liu, A. J. Gu, G. Z. Liang, Q. B. Guan, and L. Yuan, "Preparation and properties of novel high performance UVcurable epoxy acrylate/hyperbranched polysiloxane coatings," Progress in Organic Coatings, vol. 74, no. 1, pp. 142-150, 2012.

[4] D. M. Mahli, M. J. Steffenhagen, L.-L. Xing, and J. E. Glass, "Surfactant behavior and its influence on the viscosity of associative thickeners solutions, thickened latex dispersions, and waterborne latex coatings," Journal of Coatings Technology, vol. 75, no. 938, pp. 39-51, 2003.

[5] S. X. Ma and S. L. Cooper, "Shear thickening in aqueous solutions of hydrocarbon end-capped poly(ethylene oxide)," Macromolecules, vol. 34, no. 10, pp. 3294-3301, 2001.

[6] M. Barmar, M. Barikani, and B. Kaffashi, "Steady shear viscosity study of various HEUR models with different hydrophilic and hydrophobic sizes," Colloids and Surfaces A: Physicochemical and Engineering Aspects, vol. 253, no. 1-3, pp. 77-82, 2005.
[7] Z. P. Zhang, "The reasonable collocation of associative thickener with dispersing agent in emulsion paints," Modern Paint \& Finishing, no. 3, pp. 46-48, 2005.

[8] Z. Liu, J. Q. Xu, and H. B. Xia, "Research of polyurethane associative thickener," Paint \& Coatings Industry, vol. 36, no. 10, pp. 26-29, 2006.

[9] G. Moreno, C. Valencia, J. M. Franco, C. Gallegos, A. Diogo, and J. C. M. Bordado, "Influence of molecular weight and free NCO content on the rheological properties of lithium lubricating greases modified with NCO-terminated prepolymers," European Polymer Journal, vol. 44, no. 7, pp. 2262-2274, 2008. 

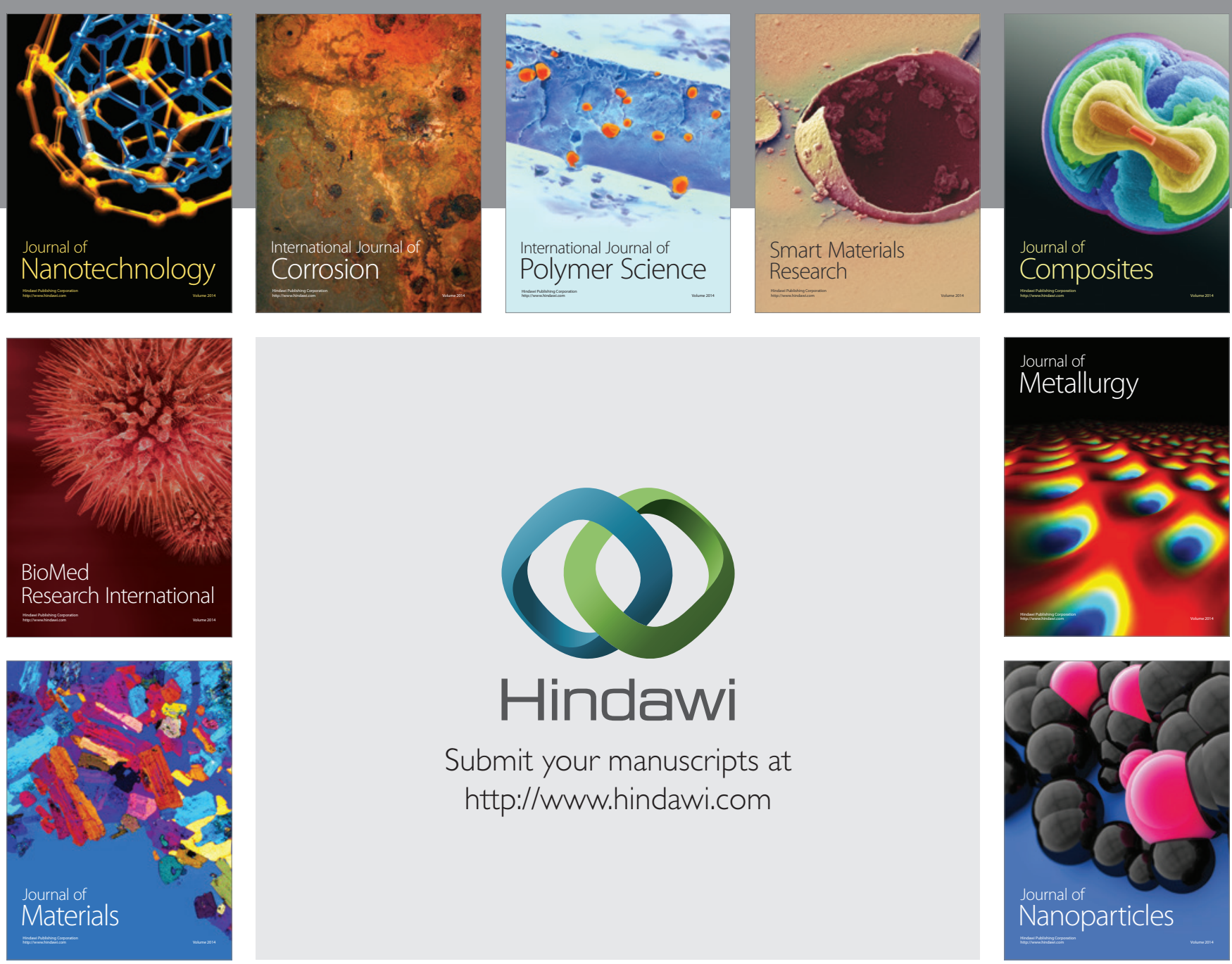

Submit your manuscripts at http://www.hindawi.com
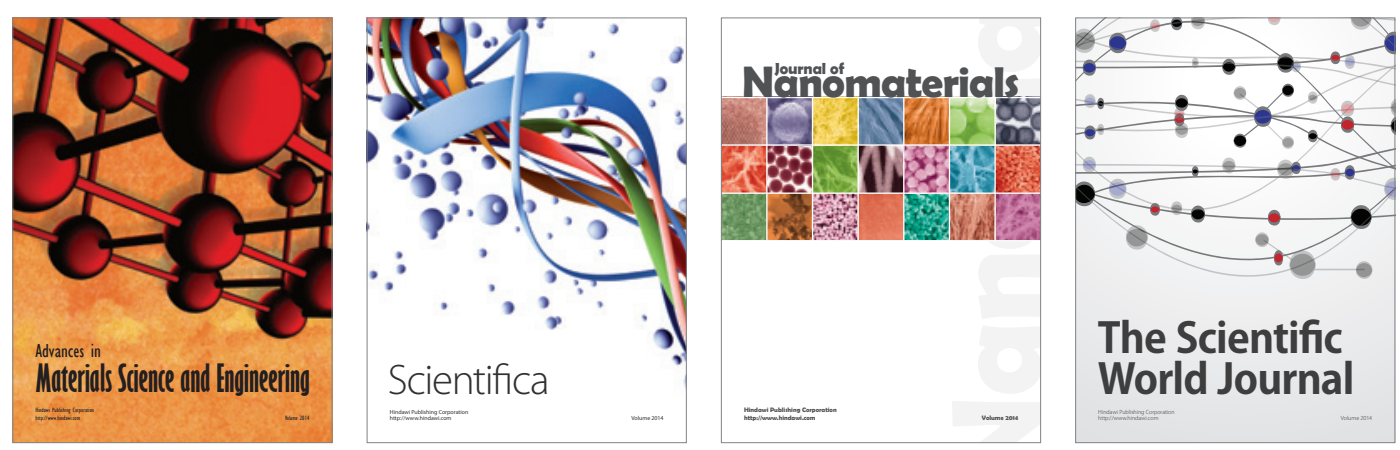

\section{The Scientific World Journal}
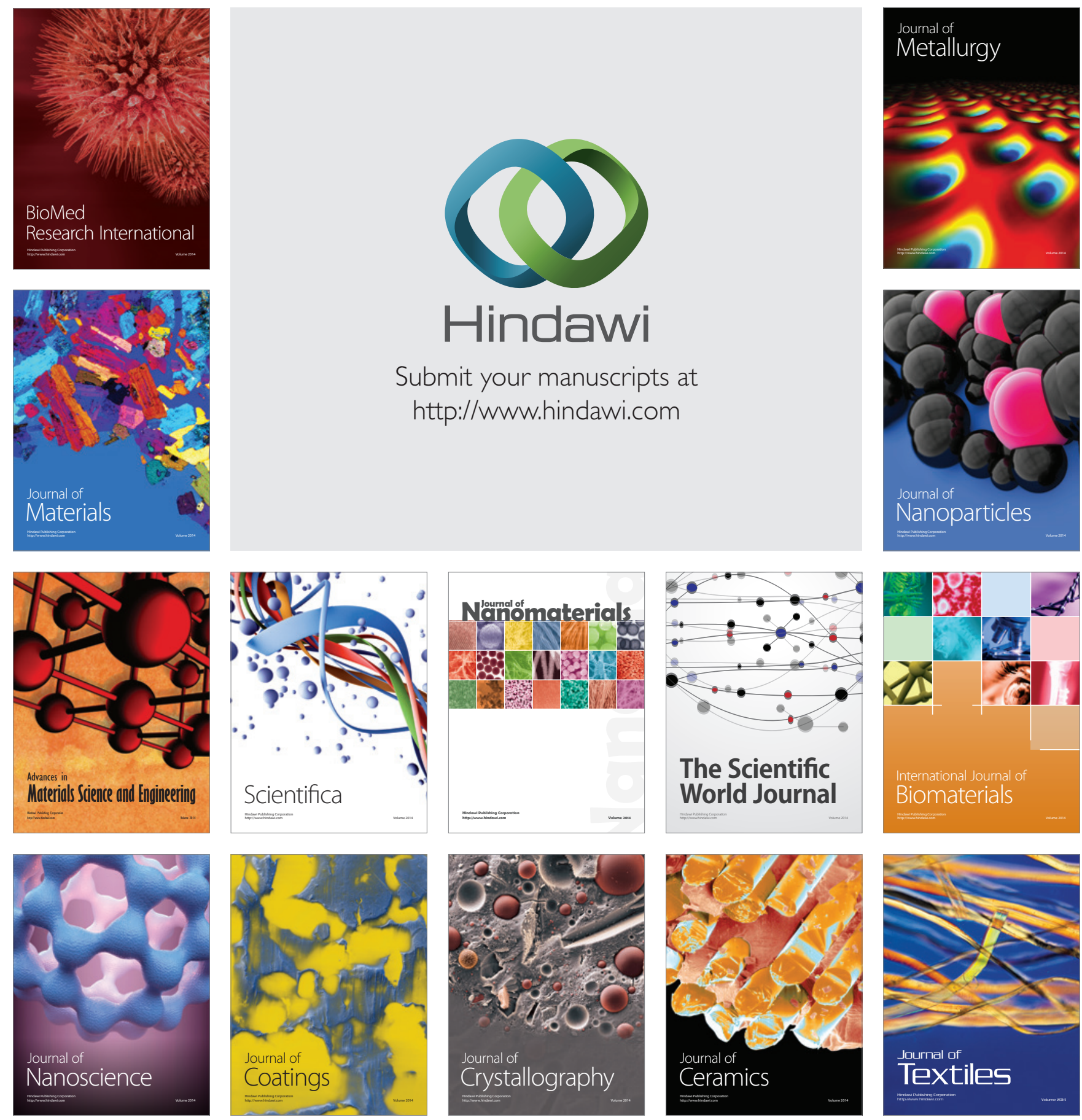